\title{
9 Resümee, Handlungsempfehlungen und Forschungsbedarfe
}

„Ich glaube, dass fast jeder Mensch in der Lage ist, sich irgendwie so weiterzuentwickeln. Und die Werkstattratsarbeit ist ein gutes Mittel dazu."

(Vertrauens-/Assistenzperson, Gruppendiskussion 1, Absatz 290)

Dieses Zitat aus den durchgeführten Erhebungen deutet die Auslegung von Werkstattratsarbeit aus Sicht einer Vertrauens-/Assistenzperson an. Diese Deutungsperspektive betont die Entwicklungsprozesse, die sich für Werkstattbeschäftigte durch die Arbeit der Interessenvertretung in eigener Sache ergeben. Die Worte lassen an das Potenzial für Selbstwirksamkeitserfahrungen denken, die mit Werkstattratsarbeit verbunden sind. So kann diese als „Instrument der Persönlichkeitsförderung und gesellschaftlichen Teilhabe insgesamt“" (Scheibner, 2000, S. 15) gedeutet werden, was den grundsätzlichen Aufgaben der WfbM entspricht ( 2219 Abs. 1 SGB IX). Wie bei der Theorie der betrieblichen Mitbestimmung (Abschnitt 2.2) sind unterschiedliche Lesarten von Werkstattratsarbeit möglich.

In der vorliegenden Arbeit wurde die Annäherung über die betriebliche Mitbestimmung, unter Reflexion rechtssoziologischer Annahmen und mit einem breit angelegten empirischen Zugang vorgenommen. Diese Vorgehensweise wurde gewählt, da Werkstatträte in Anlehnung an Betriebsräte entstanden sind, Werkstattratsarbeit verordnungsrechtlich stark reglementiert ist und kaum empirisches Wissen über die Umsetzung der rechtlichen Vorgaben vorhanden war. Geleitet ist die vorgelegte Arbeit von den Zielen, die rechtlichen Grundlagen der Werkstattratsarbeit und die Erfahrungsperspektive der Praxis zu verbinden und empirisch-subsumierend die Beteiligung der Werkstattbeschäftigten bzw. Werkstattratsarbeit in den fachlichen Diskurs zur Partizipation einzuordnen. Um diese Ziele pointiert zu bündeln, werden in diesem Resümee die Fragen reflektiert, die diese Arbeit eröffneten. ${ }^{162}$ Abschließend werden verschiedene Handlungsempfehlungen gegeben und Forschungsbedarfe aufgezeigt, die sich aus der Arbeit ableiten lassen.

\footnotetext{
162 Die in der Einleitung der Arbeit skizzierten übergeordneten Forschungsfragen der Arbeit wurden bereits in dem vorangegangenen Kapitel 7 anhand der detailreichen empirischen Ergebnisse beantwortet.
}

(C) Der/die Autor(en) 2022

V. Schachler, Partizipation durch Werkstatträte, Beiträge

zur Teilhabeforschung, https://doi.org/10.1007/978-3-658-35383-4_9 


\section{Wozu gibt es Werkstatträte in WfbM?}

Werkstatträte gibt es aufgrund eines besonderen Rechtsverhältnisses der Werkstattbeschäftigten in WfbM (Abschnitt 3.2). Denn das SGB IX charakterisiert das dortige Beschäftigungsverhältnis der Menschen mit Behinderungen als ein ,arbeitnehmerähnliches“, sodass die Regelungen aus dem BetrVG nicht greifen. Mit diesem Status können Werkstattbeschäftigte zu ihrer Vertretung keine Betriebsräte, keine Personalräte und keine Mitarbeitervertretung wählen. Stattdessen sieht der Rechtsanspruch auf Mitwirkung in Werkstätten vor, dass Werkstatträte gewählt werden. Diese Regelung wurde erst 1996 gefunden, 2001 mit der WMVO bzw. ergänzend dazu mit den kirchlichen Verordnungen genauer ausgestaltet und Ende 2016 auf einen Mitbestimmungsanspruch erweitert (Abschnitt 3.3).

\section{Wie charakterisieren sich die Arbeitsbeziehungen in WfbM?}

Unter dem Begriff der Arbeitsbeziehungen werden in der Arbeitswelt die (organisierten) Beziehungen zwischen den dortigen Akteuren, deren Charakteristiken und Austauschverhältnisse thematisiert (Abschnitt 2.2). Bezogen auf die Arbeitsbeziehungen in WfbM (Abschnitt 3.1) sind Werkstattbeschäftigte zum einen Leistungsnutzende mit einem Rechtsanspruch auf einen Arbeitsplatz in WfbM, zum anderen Arbeitnehmer*innen mit (abgeschwächten) arbeitnehmerähnlichen Rechten. Das Verhältnis zwischen Werkstatt und Werkstattbeschäftigten ist so angelegt, dass bei Vorliegen der entsprechenden Kriterien ein Arbeitsplatz bereitgestellt werden muss. Die WfbM finanzieren sich über Leistungsentgelte, die sie für die Bereitstellung der Werkstattplätze und die Erfüllung der gesetzlich definierten Ziele bekommen: Teilhabe zu ermöglichen und Menschen mit Behinderungen bestmöglich zu fördern. Die wirtschaftliche Erwerbstätigkeit gesellt sich sekundär hinzu, obwohl WfbM nicht ohne diese auskommen können und Werkstattbeschäftigte ein Mindestmaß an wirtschaftlich verwertbarer Arbeitsleistung erfüllen müssen (Abschnitt 3.1.2). Bezogen auf den Sinn und Zweck der WfbM ist die Mitwirkung und Mitbestimmung der Werkstattbeschäftigten damit noch bedeutsamer als im privatwirtschaftlichen Bereich, wo diese dem Ausgleich der tendenziell asymmetrischen Arbeitsbeziehungen zwischen Beschäftigten und Arbeitgeber dient, ohne dass hier ein Auftrag des Arbeitgebers zur Persönlichkeitsentwicklung gegeben ist. Für WfbM kann jedoch nicht davon ausgegangen werden, dass das Beziehungsgefüge als Leistungsnehmer*in (Werkstattbeschäftigte) und Anbieter von Teilhabeleistungen (Werkstatt/-personal) im Alltag per se erfahrbar ist. Vielmehr gibt es Hinweise darauf, dass zwischen den Personengruppen der 
Menschen mit regulärem und mit arbeitnehmerähnlichem Rechtsverhältnis eine Machtasymmetrie zu Ungunsten der Werkstattbeschäftigten besteht (Abschnitt 3.1.3).

Wie arbeiten Werkstatträte und gelingt es ihnen, ihre Rechte zu mobilisieren?

Wie Werkstatträte arbeiten, wird in den Kapiteln 6 bis 8 ausführlich dargestellt und analysiert. Bezogen auf ihre Rechtsmobilisierung kann festgehalten werden, dass es Werkstatträten teilweise gelingt, ihre Rechte als „law in action“ einzufordern. Ein Teil der Werkstatträte nutzt die Widerspruchsmöglichkeit, ein anderer Teil noch nicht. Prekär sind die Ergebnisse zu den Vermittlungsstellen. Dass diese nur in jeder dritten Werkstatt eingerichtet sind, schmälert die Einfluss- und Durchsetzungsmöglichkeiten der Gremien. Hier haben Werkstatträte ihre Rechte noch nicht mobilisiert.

Der Befund zu den Vermittlungsstellen lässt sich so interpretieren, dass die Reichweite der Mitbestimmung von Werkstatträten in Werkstätten noch nicht konsequent gedacht wird. Konfliktfälle sind gewissermaßen die „Nagelprobe für jeden Interessensvertretungsprozeß“ (Breit \& Kotthoff, 1990, S. 49). Ein funktionierendes Verfahren im Streitfall ist als ,juristischer Faktor der Rechtsmobilisierung“ (Baer, 2017, S. 226) zentral. Auf dieses Verfahren müssen Werkstatträte ohne viele Unklarheiten zurückgreifen können. Wenn die Einrichtung einer Vermittlungsstelle erst im Konfliktfall angegangen wird, ist nicht garantiert, dass die Beteiligungsrechte des Werkstattrats wie vorgesehen eingehalten werden. Dazu sind Werkstatträte noch zu sehr auf ein kooperatives Verhalten der Werkstattleitung angewiesen. Allerdings scheint in einem Teil der Werkstätten die Umstellung der Mitwirkungs- auf Mitbestimmungsrechte des Werkstattrats 2017 bereits etwas bewirkt zu haben. Zumindest lässt sich das Ausmaß der erst kürzlich eingerichteten Vermittlungsstellen so interpretieren.

\section{Kann sich in einem separierenden System Partizipation vollziehen?}

Grundsätzlich kann sich Partizipation auch in einem Sondersystem vollziehen. Allerdings sind die (Arbeits-)Beziehungen zwischen den dortigen Akteuren durch die gegebenen Bedingungen geprägt, wie dies in den vorangegangenen Ausführungen zu den Arbeitsbeziehungen in WfbM thematisiert wurde. Ein System, das als separierend gilt, kann für den Vollzug von Partizipation tendenziell hemmend sein. Jedoch hängt die Antwort auf die Frage, ob eine Aktivität, ein Instrument, eine Handlung etc. als Partizipation eingestuft wird, ganz davon ab, welches Partizipationsverständnis zugrunde gelegt wird und ob theoretische Bedingungen oder ein praktischer Handlungsvollzug betrachtet werden. So kann die Frage nach der 
Partizipationseinstufung bei einem Sachverhalt sehr unterschiedlich ausfallen. Im Folgenden wird eine Einstufung zunächst de jure nach den Verordnungsvorgaben unternommen.

Kann der Beteiligungsanspruch in WfbM nach den Vorgaben der WMVO als Partizipation bezeichnet werden?

Nach dem Verständnis von Scheu und Autrata (Abschnitt 2.1.1) handelt es sich bei Werkstatträten als formalisiertes Gremium zur Beteiligung der Werkstattbeschäftigten und deren Interessenvertretung nicht um Partizipation. Die Aufgaben des Gremiums und der Gegenstandsbereich des Handelns sind mit der WMVO festgelegt. Inwieweit subjektive Bestimmungen der Werkstattratsmitglieder oder der Werkstattbeschäftigten als Wählerbasis mit den Verpflichtungen des Gremiums übereinstimmen, kann sich nur auf Individualebene entscheiden. Wird hingegen ein Partizipationsverständnis von Stufenleitern angewandt (Abschnitt 2.1.2) und die Reichweite der mit der WMVO vorgegebenen Rechte von Werkstatträten betrachtet, ist das Maß der Beteiligung in ausgewählten Bereichen als Partizipation einzustufen. De jure ist mit den Mitbestimmungs- und Mitwirkungsrechten eine Machtteilnahme der Werkstattbeschäftigten an Entscheidungen über das Gremium des Werkstattrats verbindlich vorgegeben. Es hat eine Machtabgabe der Entscheidungsgewalt der Werkstattleitung zu erfolgen. Dies ist eine Pflicht und kann nicht einseitig verändert werden.

Um die demokratischen Partizipationsqualitäten des Beteiligungsinstruments zu bewerten, lassen sich die folgenden drei Maßstäbe von Kißler et al. (2011, S. 41) konsultieren:

1. Die „Regelproduktion“ - woraus geht das Verfahren hervor?

Als Gremium der Interessenvertretung der Werkstattbeschäftigten sind Werkstatträte nach dem SGB IX in WfbM einzurichten. Dies ist zwar keine Pflicht, jedoch haben die Werkstätten dies als fachliche Anforderung zu ermöglichen ( $\$ 14$ WVO). Mit wenigen Ausnahmen sind die Gremien auch vorhanden, das zeigt die empirische Analyse. Eine solch flächendeckende Verbreitung einer Interessenvertretung ist im Bereich von Arbeit und Beschäftigung keine Selbstverständlichkeit. Betriebsräte im privatwirtschaftlichen Bereich sind weitestgehend dem Engagement der Arbeitnehmer*innen überlassen. Der Verbreitungsgrad in der Privatwirtschaft ist eher gering und wird mit $<10 \%$ für Betriebe $\mathrm{ab}$ fünf Beschäftigten beziffert (Ellguth \& Kohaut, 2019, S. 295). Die „Regelproduktion“ (Kißler et al., 2011, S. 41) von Werkstatträten ist demnach regelmäßig gegeben und als hoch einzustufen. 
2. Die „Partizipationsquote“ - wer hat das Recht, am Verfahren teilzunehmen?

Anders ist hingegen die „Partizipationsquote“ (ebd.), die nach Kißler et al. auch für Betriebsräte gering ausfällt (siehe Abschnitt 2.2.4). Der Werkstattrat ist eine mittelbare Beteiligungsform. Gewählte Vertreter*innen sollen im Sinne der Werkstattbeschäftigten Einfluss auf das Werkstattgeschehen nehmen. Das Gremium ist keine unmittelbare Beteiligung am Arbeitsplatz der Werkstattbeschäftigten; nur die gewählten Personen sind legitimiert, im Rahmen ihrer Befugnisse zu partizipieren.

3. Der „Partizipationsgrad“ - beeinflusst das Verfahren Entscheidungen?

Als drittes Merkmal hat der „Partizipationsgrad“ (Kißler et al., 2011, S. 41) der Werkstattbeschäftigten in WfbM ein mittleres Niveau. In den definierten, eher lebensweltlichen Bereichen können Werkstatträte verbindlich mitwirken und mitbestimmen und damit Einfluss auf Entscheidungen nehmen.

Ungeachtet aller Kritik an WfbM ist damit nach Maßstäben, die an demokratische Partizipationsverfahren angelegt werden, die Qualität der Beteiligung in WfbM vergleichsweise hoch. Zumindest im Vergleich zu anderen Beteiligungsinstrumenten und - de jure - gemäß den formalen Vorgaben.

Kann die derzeitige Einlösung des Beteiligungsanspruchs in WfbM nach den empirischen Ergebnissen als Partizipation bezeichnet werden?

Als Arbeitsdefinition wurde festgehalten, dass es bei Partizipation im Kern um eine auf Egalität gerichtete Einflussnahme geht (Abschnitt 2.3). Partizipation beschreibt ein aktives, prozesshaftes, an demokratischen Qualitäten orientiertes, einflussnehmendes und machtvolles Handeln. Es geht um die Gestaltung von und die Teilhabe an Entscheidungen. In den qualitativen Ergebnissen zeichnet sich ab, dass sich viele Werkstatträte noch nicht in einer Verhandlungsposition befinden, in der solch ein Handeln möglich ist, und dass es noch nicht zu einer gestaltenden Entscheidungsteilhabe dieser Gremien kommt. Vereinzelt präsentieren sich Werkstattratsmitglieder demgegenüber in einer machtvollen Position oder berichten von einem mittlerweile egalitäreren Verhältnis (Abschnitt 6.1.3 und 6.1.4). Gemessen an den Indikatoren einer abgeschlossenen Betriebsvereinbarung und eines eingelegten Widerspruchs, die als manifeste Variablen einflussnehmendes Handeln und die Machtteilhabe an Entscheidungen symbolisieren können, wurde eine Typologie der Werkstatträte erstellt (Abschnitt 7.2.2). 
Knapp jeder fünfte Werkstattrat entspricht demnach dem einflussnehmenden, durchsetzungsaffinen Werkstattratstyp, der mindestens auf eine abgeschlossene Betriebsvereinbarung und einen eingelegten Widerspruch in Angelegenheiten der Mitwirkung und Mitbestimmung in den letzten zwei Jahren verweisen kann. Auch wenn die weiteren Umstände der tatsächlichen Einflussnahme und Machtteilhabe offenbleiben, gelingt es diesen Werkstatträten, ihre Befugnisse in konkrete Handlungen zu überführen, sie werden in Entscheidungen einbezogen und haben Gestaltungsoptionen. Ihr Handeln zeigt partizipative Züge. Demgegenüber entsprechen rund $40 \%$ der Werkstatträte dem ignorierten Werkstattratstyp. Dieser Werkstattrat ist zwar eingerichtet, nimmt aber auf das Betriebsgeschehen keinen Einfluss. Eine Partizipation dieser Gremien ist nicht feststellbar.

Als weiteres grundlegendes Element von Beteiligung lässt sich die Informationsübermittlung anführen. In Stufenmodellen wird sie als Vorstufe der Partizipation (Wright et al., 2010) oder symbolische Beteiligungsform (Arnstein, 1969) angegeben. Mit den empirischen Ergebnissen zeigt sich, dass mindestens in einem Drittel der Werkstätten die Informationsübermittlung an den Werkstattrat nicht adäquat erfolgt. Solange dies nicht gegeben ist, kann nicht davon gesprochen werden, dass weitergehende Beteiligungsformen oder gar Partizipation in $\mathrm{WfbM}$ ermöglicht sind. Insgesamt ist es somit für viele WfbM derzeit noch angemessener, lediglich von Beteiligungsmöglichkeiten zu sprechen, als diese per se als Partizipation auszugeben.

Auf dem Weg zur Partizipation spielen verschiedene Aspekte eine Rolle. Es gibt ein Konglomerat von Faktoren, die beteiligt sind, wenn ein aktiver und autonomer Werkstattrat entsteht. In der statistischen Analyse zeigen sich die dauerhafte Freistellung der*des ersten Vorsitzenden, der Unterstützungsumfang der Vertrauensperson(en), eine abgesprochene Aufgabenverteilung im Werkstattrat und die Anzahl der Werkstattratsmitglieder als einflussnehmende Faktoren, an denen bewusst angesetzt werden kann. So kann einerseits - vonseiten der Werkstatt - der Unterstützungsumfang der Vertrauensperson etwa durch klare und verbindliche Rahmenbedingungen gefördert (Abschnitt 7.1.1.9 und 7.1.1.10) oder die Anzahl der Werkstattratsmitglieder auf freiwilliger Basis erweitert werden. Andererseits können Werkstatträte durch eine bewusste Beschäftigung mit ihrer Arbeitsweise oder durch eine Einforderung ihrer Ansprüche dazu beitragen, dass Partizipation entsteht. Statistisch zeigt sich, dass das Aktivitätsniveau und die autonome Arbeitsweise eines Werkstattrats korrelieren. Die Förderung der Autonomie eines Werkstattrats, etwa durch die Bereitstellung eines eigenen Büros, 
wirkt sich förderlich auf das Aktivitätsniveau eines Werkstattrats aus. Gleichfalls trägt ein höheres Aktivitätsniveau zu einer autonomeren Arbeitsweise bei.

\section{Handlungsempfehlungen und Forschungsbedarfe}

Partizipation - Begriffsdifferenzierung im Zusammenhang der Themen von Menschen mit Behinderungen

Partizipation ist als demokratische Leitlinie in unterschiedlichen Kontexten gängig, so auch in dem Fachdiskurs zu Behinderung, wenngleich hier eher eine Gleichsetzung mit dem Teilhabebegriff als eine eigenständige Betrachtungsweise anzutreffen ist (Abschnitt 2.1.3). Mit der herausgearbeiteten Arbeitsdefinition lässt sich aufzeigen, dass mit Partizipation als einer auf Egalität gerichteten Einflussnahme an Entscheidungsprozessen etwas anderes gemeint ist als die Inhalte, die unter dem Begriff der Teilhabe von Menschen mit Behinderungen verhandelt werden (Abschnitt 2.3). In diesem Sinne sind die Begriffe Teilhabe und Partizipation im Kontext von Behinderung nicht bedeutungsgleich. Beizupflichten ist Hirschberg (2010), die fordert, dass in diesem Bereich „,der Begriff ,Partizipation“ auch in die deutschsprachige Diskussion aufgenommen werden“ (S. 2) muss. Damit Partizipation nicht als bloßes Beiwerk des Teilhabebegriffs erscheint, ist dringlich zu empfehlen, sich darüber zu verständigen, von welchen Vorstellungen ausgegangen wird, bevor Möglichkeiten oder Handlungen als Partizipation von Menschen mit Behinderungen ausgegeben werden.

\section{Einrichtung von Vermittlungsstellen und Erweiterung der Strategien zur Rechtsmobilisie-} rung

Wie beim BetrVG ist für die WMVO keine „staatliche Kontrollinstitution“ (Blankenburg, 1977, S. 49) zur Einhaltung vorgesehen. Neben der Anrufung der Vermittlungsstelle bezieht sich für Werkstatträte die weitergehende Rechtsdurchsetzung auf die Hinzuziehung des Arbeitsgerichts (Bernzen et al., 2020, S. 57). Diese Hinzuziehung ist sehr voraussetzungsvoll und scheint eine größere Hürde als die Einrichtung einer Vermittlungsstelle zu sein. Nach Breit und Kotthoff (1990) ist die „Wirksamkeit probater Konfliktregulierungsverfahren ... der eigentliche Sinn der Mitwirkung“ (S. 49). Solange also Vermittlungsstellen fehlen und alternative Verfahrenswege der Konfliktregulierung nicht klar und zuverlässig greifen, bleibt fraglich, ob die Mitwirkungs- und Mitbestimmungsrechte des Werkstattrats in WfbM konsequent möglich und gegeben sind. So sind Werkstätten aufgefordert, die Einrichtung von Vermittlungsstellen vorausschauend anzugehen. Gleichzeitig können sie damit eine Öffnung im Sinne 
ihrer propagierten Weiterentwicklung zum Ausdruck bringen. Durch den Gesetzgeber sollte ergänzend dazu eine Klarstellung in dreierlei Hinsicht erfolgen: erstens, ob die Vermittlungsstelle nun als Dauer- oder anlassbezogene Instanz gedacht ist, zweitens, wie sichergestellt werden kann, dass die Mitbestimmungs- und Mitwirkungsrechte im Konfliktfall garantiert sind, wenn noch keine Vermittlungsstelle besteht, und drittens, an wen sich die Frauenbeauftragten in dieser Situation wenden können. Werkstatträten lässt sich demgegenüber empfehlen, ihre Strategien zur Rechtsmobilisierung zu erweitern.

Rechtsmobilisierung ist ein selbstermächtigender, aktiver Prozess (Abschnitt 4.3), so wie sich dies gleichfalls für Partizipation festhalten lässt. Für den Werkstattrat vor Ort kann dies bedeuten, seine Argumentationslinie mit Schulungen oder Rechtsberatung (bezahlt über das selbst verwaltete Budget) einzuüben, die Errichtung einer Vermittlungsstelle einzufordern und damit auch sein Netzwerk zu erweitern oder die Verfahrenswege zumindest abzuklären, damit die Mitbestimmungs- und Mitwirkungsrechte kein „schlafendes Recht“, ein „law on the books“" (Baer, 2017, S. 226) bleiben.

Für die überregionalen Interessenvertretungen können mögliche Strategien in der Wissensvermittlung liegen, wie dies von Werkstatträte Deutschland mit der Veröffentlichung der Ergebnisse einer Rechtsberatung (Veit, 2020) oder mit Handlungsempfehlungen zur Vermittlungsstelle (Werkstatträte Deutschland \& BAG WfbM, 2020) bereits praktiziert wird. Mobilisierungsstrategien könnten aber auch darin liegen, Möglichkeiten zur diskursiven Wissensanwendung zu geben, niedrigschwellige Wege zur Rechtsberatung aufzuzeigen oder im Sinne einer strategischen Prozessführung arbeitsgerichtliche Abklärungen zu unterstützen und zu kommunizieren, etwa in Streitfällen der angemessenen Ausstattung des Werkstattrats.

\section{Drei Mitwirkungsverordnungen - Begegnung der verkomplizierten Sachlage}

Mit der WMVO, der Caritas-WMO und der DMVO bestehen drei im Wortlaut und in Detailaspekten unterschiedliche Mitwirkungsverordnungen. Dies verkompliziert die Sachlage in einem Bereich, der auf eine vereinfachte Sprachwahl angewiesen ist. Für die Kommunikation in einfacher oder Leichter Sprache, für den persönlichen Austausch von Werkstattratsmitgliedern, von Vertrauenspersonen und von Werkstattleitungen untereinander, für die Schulungsmöglichkeiten und -themen sowie für die überregionale Gremienarbeit ist dies sehr unvorteilhaft.

Die Existenz von drei Verordnungen wurde in der ersten WMVO-Reform 2016 aufgrund von Protesten der Interessenverbände beibehalten. Proteste wurden u. a. von der Diakonie Deutschland und dem Bundesverband evangelische Behindertenhilfe sowie dem Deutschen 
Caritasverband e. V. geäußert (Schachler \& Schreiner, 2017, S. 3). Angesichts dieser Beibehaltungsinitiativen bleiben diese Verbände aufgefordert, sich besonders zu engagieren, um den aus drei Verordnungen entstehenden Nachteilen $\mathrm{zu}$ begegnen, auch wenn die in $\S 118$ Abs. 2 BetrVG verankerten Sonderrechte für Tendenzbetriebe Gültigkeit besitzen.

\section{Reformnotwendigkeiten: Zweigwerkstatträte, verbindliche Initiativ- und weitere Mitbestim- mungsrechte}

Ein Vorteil der kirchlichen Verordnungen ist, dass diese die Möglichkeit zur Bildung von Zweigwerkstätten explizit benennen (Abschnitt 3.3.3). Dies ist für den Beteiligungsanspruch der Werkstattbeschäftigten sehr begrüßenswert. Betriebsstätten einer Werkstatt liegen räumlich oft weit voneinander entfernt, mit einer schlechten öffentlichen Verkehrsanbindung. Ein Zweigwerkstattrat kann demgegenüber vor Ort aufgesucht werden, ist vor Ort aktiv und bekannt und hat so einen besseren Zugang zu den Werkstattbeschäftigten und eine größere Nähe zu deren Themen als ein einziger Werkstattrat für viele Betriebsstätten mit räumlicher Distanz. Es ist auf die Wiedereinführung von Zweigwerkstatträten für Werkstatträte zu plädieren (so bereits Wendt, 2002, S. 323). Die explizite Benennung sollte wieder in die WMVO aufgenommen werden. Zu bedenken ist in diesem Zusammenhang, dass die Mitgliederzahlen des Werkstattrats trotz der Erhöhung im Jahr 2016 gegenüber dem BetrVG immer noch nachteilig sind.

Angesichts der großen Bedeutung der Mitwirkungs- und Mitbestimmungsmöglichkeiten der Werkstattbeschäftigten in WfbM im Sinne einer selbstbestimmten Leistungserbringung und für ein plausibles Werkstättensystem ist zu bedenken, dass die derzeitigen Rechte der WMVO noch Erweiterungspotenziale bieten. Eine mögliche Erweiterung läge bspw. in verbindlichen Initiativrechten des Werkstattrats. So schlug bereits Naendrup Mitte der 1980erJahre ein Initiativrecht für Kündigungen von Dienstverträgen vor, wenn ein Vertrauensverhältnis zu einem*r Mitarbeitenden der Einrichtung nachhaltig zerstört ist (ebd., 1984, S. 275 f., S. 298). Um Gewalt- und Missbrauchsfällen in WfbM effektiv zu begegnen, sollte dieser Vorschlag diskutiert werden, und gleichfalls ein Zustimmungsrecht bei allen Personalentscheidungen, die die Vertrauensperson betreffen, sodass diese nicht einseitig durch die Werkstatt versetzt oder gekündigt werden kann.

\section{Sicherstellung einer ausreichenden Finanzierung und personellen Unterstützung}

Daneben sind eine ausreichende Finanzierung und personelle Unterstützung des Werkstattrats sicherzustellen. Kritische Stimmen zur unterfinanzierten pädagogischen Personalsituation in 
WfbM (Greving \& Scheibner, 2014, S. 39 ff.) sind ernst zu nehmen. Das Schlüsselverhältnis in WfbM wird sowohl für die Begleitenden Dienste als auch für die Fachkräfte zur Arbeitsund Berufsförderung als unzureichend benannt (Abschnitt 3.1.2). Für die Fachkräfte zur Arbeits- und Berufsförderung (Gruppenleitungen) wird zudem das geringe pädagogische Qualifizierungsniveau kritisiert. Diese Befunde lassen sich auf die Vertrauenspersonen übertragen, die Werkstatträte unterstützen (sollen). Wie in der integrierten Datenanalyse und Interpretation dargestellt, kann oftmals nur in einem sehr geringen zeitlichen Umfang Unterstützung gegeben und von den Vertrauenspersonen nur wenig tätigkeitsbegleitende Schulung genutzt werden. Die Rahmenbedingungen ihrer Arbeit sind i. d. R. durch ihre Doppelfunktionen (Fachpersonal der Werkstatt und Vertrauensperson des Werkstattrats) problematisch (Abschnitt 8.1.3). Damit die Werkstattratstätigkeit möglich ist und auch für Werkstattbeschäftigte mit höheren Unterstützungsbedarfen eine Option bildet (Abschnitt 6.1.4.1), ist eine ausreichende personelle Unterstützung sicherzustellen und durch die Rehabilitationsträger zu finanzieren, wie auch die weitere Ausstattung der Gremien. So bleibt die Ressourcen- und Finanzierungsthematik eine der zentralen Fragen der Werkstattratsarbeit. ${ }^{163}$

\section{Verankerung der WfbM als Rehabilitationseinrichtung und Pflichtenliste für den Beteili- gungsanspruch}

Scheibner und Sackarendt (2020, S. 4) fordern eine klare Verankerung der WfbM als Rehabilitationseinrichtung im SGB IX (§ 51 SGB IX), womit sich ein Menschenbild etablieren kann, das sich verbindlich auf die leistungsberechtigten Beschäftigten und nicht auf die wirtschaftlich verwertbaren Produktions- und Arbeitsergebnisse ausrichtet (siehe auch Greving \& Scheibner, 2014, S. 44). In diesem Sinne empfiehlt sich eine Pflichtenliste für den Werkstattträger (verankert in $\S 222$ SGB IX), die bindend sicherstellt, dass Werkstatträte bzw. Werkstattratsmitglieder ihr Amt wahrnehmen können. Lediglich auf die Ermöglichungspflicht der Werkstatt (§ 14 WVO) und eine vertrauensvolle Zusammenarbeit mit dem Werkstattrat ( 88 WMVO) zu verweisen ist für den Werkstättensektor nicht ausreichend. ${ }^{164}$

Nicht förderlich ist in diesem Kontext, dass für Amtsbehinderungen von Werkstatträten „keine unmittelbaren Rechtsfolgen“ (Bernzen et al., 2020, S. 197) vorgesehen sind.

163 Im Rahmen der vorliegenden Arbeit wurde diese nur am Rande behandelt. Für weitere Befunde lässt sich auf Schreiner und Schachler (2021) verweisen.

${ }^{164}$ Für diesen Hinweis danke ich U. Scheibner (persönl. Mitteilung, 31.10.2018). 
Stattdessen sollen die „Rehabilitationsträger etwaigen Vollzugsdefiziten im Zusammenhang mit der Mitwirkung und Mitbestimmung entgegenwirken“ (ebd.). Doch wie erfahren sie davon? Und welche Sanktionen ergeben sich daraus, die im Interesse der Werkstattbeschäftigten liegen (Abschnitt 3.3.2)?

Eine weitere Schwachstelle der WMVO liegt in der einseitigen Reduktion der Mitbestimmungsrechte bei Überschneidungen mit Angelegenheiten des Betriebsrats / der MAV / sonstigen Arbeitnehmervertretungen auf einvernehmliche Lösungen ohne Hinzuziehung der Vermittlungsstelle. Dies kann die Stellung des Werkstattrats weiter schwächen (Abschnitt 3.3.2) und muss weiter beachtet werden.

\section{Umsetzung eines teilhabeorientierten Dienstleistungsverständnisses}

Unabhängig von allen rechtlichen Vorgaben lässt sich festhalten, dass Werkstätten, die den Mehrwert eines starken Werkstattrats erkennen und die Stärkung des Gremiums als strategische Managementform begreifen, den klaren Vorteil haben, sich einem modernen, teilhabeorientierten Dienstleistungsverständnis im Sinne der Anerkennungsperspektive der UN-BRK und der Zielrichtung der Personenzentrierung nach dem SGB IX anzunähern. Die durchgeführten Untersuchungen zeigen, dass dies noch nicht bei allen Werkstätten eine Selbstverständlichkeit ist.

In diesem Zusammenhang ist darauf zu verweisen, dass Mitbestimmung und Mitwirkung in WfbM sowie die Regelung und inhaltliche Gestaltung der Zusammenarbeit von Werkstattleitung und Werkstattrat zu den Qualitätsansprüchen von WfbM gehören. Dementsprechend sollten diese Themen in Qualitätshandbüchern und -systemen verankert werden. ${ }^{165}$

\section{Einbindung der Werkstattbeschäftigten in die Zukunftsdiskussion der WfbM}

Ein Rückbau des Werkstättensystems findet bis dato noch nicht statt. Falls dies künftig der Fall ist oder konkretere Weiterentwicklungen des Werkstättensystems angegangen werden, bestände dringender Anlass dazu, weitergehende Mitbestimmungsrechte zu diesen Aspekten in der WMVO zu verankern. Ferner ist im Zuge der Diskussionen der WfbM im Hinblick auf Artikel 27 UN-BRK (Abschnitt 3.1.4) und der zukünftigen Teilhabeleistungen im Bereich von Arbeit und Beschäftigung eine Einbindung der Werkstattbeschäftigten unerlässlich und notwendig, auch um die zur Werkstatt bestehenden alternativen Leistungsformen bekannt zu

\footnotetext{
165 Für diesen Hinweis danke ich Dr. W. Schlummer (persönl. Mitteilung, 08.12.2020).
} 
machen. Die Einbindung sollte auf lokaler, regionaler und überregionaler Ebene in Zusammenarbeit mit den entsprechenden Ansprechpartner*innen und Verbänden erfolgen. Auf bundesweiter Ebene tritt das politische Engagement von Werkstatträten bereits deutlich hervor. Auch auf lokaler Ebene können Werkstatträte ein Engagement entwickeln, sich in ihr direktes Umfeld einbringen und damit Synergien zu den künftigen örtlichen beruflichen Teilhabeangeboten erzeugen.

\section{Beachtung der Werkstattratsarbeit unter Corona-Bedingungen}

Corona-Maßnahmen fallen in den Mitbestimmungs- und Mitwirkungsbereich des Werkstattrats. Sie betreffen u. a. den Gesundheitsschutz, die Gestaltung von Arbeitsplätzen, das Verhalten im Arbeitsbereich und die Arbeitszeiten. Dies sind Themen, für die Mitbestimmungsund Mitwirkungsrechte der Werkstatträte bestehen. Offen ist, ob und wenn ja, wie ein Einbezug der Werkstatträte im Zuge der Maßnahmen im Umgang mit COVID-19 erfolgt (ist). An dieser Stelle zeigt sich weiterer Forschungsbedarf. So kann die Einbindung in Krisenstäbe (Berg, 2020) und der Umgang mit den Gremien der Interessenvertretung in Krisenzeiten Auskunft über Wertigkeiten und (nicht) funktionierende Abläufe offenbaren.

\section{Aufgreifen weiterer Forschungsbedarfe}

Die vorliegende Studie zeichnet nach rund zwanzigjährigem Bestehen der WMVO ein erstes, umfassendes Bild, wie die Umsetzung des Beteiligungsanspruchs in WfbM jenseits der rechtlichen Vorgaben tatsächlich ausfällt. Die dezidierten empirischen Ergebnisse und die Erarbeitung einer bundesweiten Datenlage zu umfänglichen Aspekten der Werkstattratsarbeit mit Bezug auf die rechtlichen Gegebenheiten und die empirische Mitbestimmungsforschung sind deren besondere Stärke. Zugleich sind damit Limitationen verbunden. Andere, nicht fokussierte Lesarten von Werkstattratsarbeit lägen bspw. darin, diese dienstleistungstheoretisch zu analysieren (Pluto, 2007, S. 37), als Element eines partizipativen Managementsystems herauszuarbeiten und mögliche Verankerungen in Qualitätshandbüchern und -managementsystem zu akzentuieren, oder Werkstattratsarbeit stärker pädagogisch zu betrachten und Bildungsprozesse bzw. Kompetenzfragen zu thematisieren, wie dies der Ansatz von Schlummer und Schütte (2006) ist. So gehen mit Zugangsentscheidungen und forschungsmethodischen Auswahlprozessen notgedrungen immer Fokussierungen einher, die mit konstruieren, was letztlich als Ergebnis und Forschungsprodukt erscheint.

In den skizzierten thematischen Limitationen der Arbeit, über die erzielten Ergebnisse und Empfehlungen deuten sich weitere Forschungsbedarfe an. Werden diese aufgegriffen, kann 
die wissenschaftliche, externe Beachtung ein Korrektiv sein, um Werkstatträte in ihrer Stellung innerhalb der WfbM zu festigen, an den primären Auftrag der WfbM zu erinnern, und damit einen Beitrag dazu leisten, dass eine Leistungserbringung im Sinne der Förderung der Selbstbestimmung und Teilhabe von Menschen mit Behinderungen (§ 1 SGB IX) erfolgt. Vorerst verdeutlicht diese Forschungsarbeit mit ihrer Fokussierung auf Partizipation vor allem zwei Dinge: zum einen, dass sich die Praxis und die Politik nicht darauf ausruhen können, dass Mitbestimmung im SGB IX und der WMVO kodifiziert ist; zum anderen, dass auch zukünftig Forschung die Praxis mit fundierten Beiträgen und Erkenntnissen unterstützen kann. 